\title{
Ein Fall von Inversio uteri im 78. Lebensjahre.
}

\author{
Von \\ Prof. Friedrich Schauta.
}

(Mit Abbildung auf Tafel II.)

Am 12. November 1891 stellte mir Herr Dr. Sonnenschein eine 78jährige Frau vor, welche seit einem Jahre das Vortreten eines Zapfens aus der Vulva bei zeitweiser Retentio urinae und mässigem Blutabgange bemerkte. Vor etwas mehr als Jahresfrist will sie zuerst beobachtet haben, dass sich hie und da aus der Scheide eine kleine Geschwulst hervordränge, über die sie jedoch weiter nichts angeben konnte, als dass dieselbe gewöhnlich während der Nacht von selbst zurückging und ihr sonst keine Beschwerden verursachte. In späterer Zeit sei diese Geschwulst immer öfter und immer grösser zum Vorscheine gekommen und vor ungefähr 2-3 Monaten sei ein mehr als apfelgrosses Stück vorgefallen, welches nicht mehr von selbst zurückging, sondern von der Kranken zurückgedrängt werden musste, jedoch immer und immer wieder nach kurzer Zeit vorfiel.' Zur selben Zeit klagte die Kranke über heftige Kreuz- und Bauchschmerzen, auch ging durch mehrere Tage Blut aus den Genitalien ab. Ausserdem theilte die Tochter der Kranken mit, dass Letztere fortwährend an der Geschwulst zog und zerrte, in der Meinung, sich von derselben auf diese Weise zu befreien. In den letzten 3 Monaten leidet die Kranke ausserdem an Urinbeschwerden, indem sich während des Urinirens ziehende Schmerzen in der Unterbauchgegend einstellten, und zwar nur während des Harnlassens.

Im Uebrigen ergab die Anamnese, dass bei der Kranken im 15. Lebensjahre zum ersten Male die Menstruation eingetreten war; dieselbe kehrte in regelmässigen Zwischenräumen von 4 Wochen wieder und dauerte gewöhnlich 4 Tage. Bis zu ihrem 20. Lebensjahre, in welchem sie zum ersten und zugleich zum letzten Male gebar, bekam sie regelmässig vor Eintritt der Periode heftige krampfartige Schmerzen im Bauche, die nach Beginn der Blutung 
aufhörten. Seit der Entbindung, welche vollkommen normal verlief, traten diese Schmerzen nur noch äusserst selten auf. Seit dem 48. Lebensjahre besteht klimakterische Amenorrhoe. In ihrem 10. Lebensjahre machte die Kranke Variola durch; sonst war sie, abgesehen von einem seit Jahren bestehenden Bronchialkatarrh, stets gesund. Sie erfreut sich trotz ihres hohen Alters einer ungewöhnlichen Rüstigkeit und war bis in allerjüngster Zeit täglich von 5 Uhr Morgens bis 7 Uhr Abends im Fragnergeschäfte ihrer Tochter thätig.

Die Untersuchung ergab folgenden Befund : Die Frau ist mittelgross, kräftig gebaut, Muskulatur schlaff, allgemeine Hautdecken blass, allenthalben in zahlreiche Falten gelegt, Temperatur 37,1, Puls 76, regelmässig, voll; Arterien starr; leichter Katarrh der Bindehäute. Im Brustsegmente der Wirbelsäule, und zwar vom 5.-10. Brustwirbel eine leichte Kyphoskoliose nach rechts. Ueber den Lungen allenthalben deutlicher Schachtelton, die Lungengrenze etwas nach abwärts gerückt; auscultatorisch lässt sich verlängertes Exspirium und vereinzeltes Giemen und Pfeifen bemerken (Emphysem und chronische Bronchitis). Herzstoss in der Mammillarlinie im 4. Intercostalraum fühl- und sichtbar. Die Herzdämpfung wegen des bestehenden Emphysems bei ganz schwacher Percussion nur annähernd feststellbar, reicht vom unteren Rande der 3. Rippe bis zur 5. Rippe und nach rechts bis zum linken Sternalrande. Die Herztöne sind rein, etwas klingend. Leber und Milz in normalen Verhältnissen; der Harn trübe, zeigt auch nach dem Filtriren eine Spur Eiweiss; Sediment bestehend aus Eiterkörperchen und einzelnen Epithelien der Harnwege; leichte Cystitis.

Aus der Scheide stülpt sich eine wurstförmige, durchaus mit Schleimhaut überkleidete Geschwulst vor, welche den Eingang um $9 \mathrm{~cm}$ überragt und eine grösste Breite von $\check{5} \mathrm{~cm}$ besitzt. Dieser Tumor lässt sich durch eine deutlich ausgeprägte weissliche Furche, welche in einer Entfernung von $7 \mathrm{~cm}$ vom peripheren Ende denselben ringförmig umgreift, deutlich in eine grössere untere und eine kleinere obere Partie abgrenzen, welche sich wesentlich von einander unterscheiden. Der untere Antheil hat eine teigig-weiche Consistenz; seine Oberfläche, die einzelne flache buckelartige Erhabenheiten zeigt, besitzt eine schmutzig-rothbraune Farbe, ist an einzelnen Stellen papillär sammtartig; zum grössten Theile zeigt sie jedoch das Aussehen einer granulirenden Fläche, blutet sehr leicht und sondert fortwährend eine schleimige, faden- 
ziehende, blutig gefärbte Flüssigkeit ab. An der Umschlagstelle der vorderen in die hintere Wand der Geschwulst, also entsprechend der unteren Kuppe, befindet sich eine fast quer verlaufende, $3 \mathrm{~cm}$ lange Furche, welche jederseits gleich weit vom Seitenrande endet und an deren beiden Enden sich je eine kleine dreieckige Oeffnung befindet. Durch die rechtsseitige Oeffnung lässt sich eine Sonde in der Richtung nach oben bis auf $15 \mathrm{~cm}$ einführen, dagegen durch die linke nur $5 \mathrm{~cm}$ tief. Unmittelbar hinter und etwas seitwärts von der rechtsseitigen Oeffnung sitzt mit einem etwa kleinfingerdicken Stiele ein etwa wallnussgrosser, runder, etwas derberer kugeliger Tumor der Geschwulst auf. Der obere Antheil des Tumors zeigt eine mehr elastische Beschaffenheit und vollständig glatte Oberfläche, deren rothe Farbe einen weissen Schimmer besitzt, als ob ein feines Häutchen oder zarter Schleier über dieselbe ausgebreitet wäre. Allenthalben an der Oberfläche sind feine Venennetze sichtbar. Dieser. Theil des Tumors setzt sich weiter in die Scheide fort und geht mit vollständig glatten Wandungen und ohne dass man eine Grenze tasten oder sehen könnte, unmittelbar in das Vaginalrohr über. Die Länge dieses in der Scheide gelegenen Uebergangstheiles beträgt an der vorderen Wand 4 , an der hinteren Wand $8 \mathrm{~cm}$. Eine in die Blase eingefühirte Sonde lässt sich nur in der Richtung nach oben vorschieben, und zwar bei gefüllter Blase auf 12, bei leerer Blase auf $81 / 2 \mathrm{~cm}$.

Der Tumor bestand also, wie sich unschwer erkennen lässt, aus der vollständig invertirten und vorgefallenen Gebärmutter und einem Theile des Scheidenrohres. Die Grenze zwischen Uterus und Cervix bildete jene vorhin beschriebene Furche. Von einem Orificium oder Orificialrande war nichts mehr zu sehen. Jene beiden Oeffnungen waren die Tubenmündungen. Das Epithel der Scheiden-, sowie das der Cervixschleimhaut war verdichtet und zeigte aus diesem Grunde jenen weisslichen Schimmer. Die Uterusschleimhaut befand sich im Zustande der chronischen Entzündung.

Mit Rücksicht auf das hohe Aiter und den ausgesprochenen Marasmus der Kranken konnte ich mich nicht sofort zur vollkommenen Exstirpation des ganzen vorgefallenen Tumors entschliessen, sondern machte vorher den Versuch, nur das Myom abzutragen, um dann eventuell durch Tamponade der Scheide oder ein entsprechendes Pessar den invertirten Uterus in der Scheide 
zurückzuhalten. Es wurde am 13. November die Entfernung des Myoms vorgenommen, und zwar indem ich eine Nadel mit doppeltem Faden durch die Basis des Myoms durchzog und die beiden Fäden nach rechts und links knüpfte und hierauf das Myom abtrug. Da aber wiederholte Versuche, den Tumor durch Tampons und Pessarien zurückzuhalten, in der Folge nicht zum Ziele führten, da die Pessarien heftige Schmerzen verursachten und Tampons von der Kranken immer wieder herausgerissen wurden, oder von selbst herausfielen, andererseits der Tumor an einzelnen Stellen bereits missfärbige Beschaffenheit zeigte und demgemäss Gangrän des invertirten Organs bei dem vorgerückten Alter der Kranken nicht ausgeschlossen erschien und die Beschwerden ausserdem sehr heftige waren, so entschloss ich mich endlich zur Radicaloperation, welche am 27. November ausgeführt wurde.

In Chloroformnarkose, welche von der Kranken ausgezeichnet vertragen wurde, wurden unmittelbar vor der früher erwähnten ringförmigen Furche (Grenze zwischen Corpus und Cervix) mit der Stielnadel drei fortlaufende Ligaturen angelegt, welche durch die ganze Dicke des Tumors hindurchgingen und bei welchen die Fäden so angelegt wurden, dass jedesmal zwei benachbarte Fäden in einem Stichkanale liefen. Es wurde also mit einer Stielnadel zunächst in der Nähe des rechten Seitenrandes sagittal durchgestochen, der Faden aus dem nahe der Spitze befindlichen Oehre entfernt und in das Oehr der Nadel ein zweiter Faden eingezogen, derselbe durch den Stichkanal zurückgeführt und die Nadel mit demselben Faden nochmals, und zwar etwa $1-1 \frac{1}{2} \mathrm{~cm}$ weiter nach links wieder sagittal in derselben Linie durchgeführt, nun der Faden aus dem Oehr entfernt und ein dritter Faden in das Oehr eingefädelt, der, mittels der Stielnadel durch den Stichkanal zurückgezogen, die dritte Ligatur darstellte. Um nun durch das nunmehrige Knüpfen der drei Ligaturen nicht die Stichkanäle auseinander zu zerren und das morsche Gewebe des senilen Uterus nicht einzureissen, wurden vor dem Schnüren die drei Ligaturen gekreuzt und dann erst geknüpft. Hierauf wurde der unterhalb der Ligatur gelegene Theil des Tumors, also der grösste Theil des invertirten Uterus, mit einem Scalpell, etwa $1 \mathrm{~cm}$ unterhalb der Ligatur amputirt. An dem Stumpfe zeigten sich mehrere grössere Gefässlumina; dieselben wurden mittels Schieber gefasst und unterbunden. Endlich wurde auch die Schnittfläche durch sieben Sagittalnähte vollkommen vereinigt, hierauf der Rumpf zurückgedrängt und mit Jodoformgaze leicht tamponirt. 
Die Kranke hatte die Narkose sehr gut überstanden; sie fühlte sich ganz wohl, der weitere Verlauf war vollkommen ungestört. Vom vierten Operationstage an brachte sie bereits zwei Stunden ausserhalb des Bettes zu und befand sich auch an den folgenden Tagen vollkommen wohl. Bei der Untersuchung am 6. December war weder von dem abgeschnürten Stumpfe, noch von den Ligaturen etwas in der Scheide nachweisbar; es hatte sich vielmehr eine Art Orificium und eine kurze Cervix gebildet, und zwar durch Reinversion des Stumpfes. Am 9. December wurde die Kranke in häusliche Pflege entlassen. Das abgestorbene Schnürstück stiess sich allmälig ab. Am 9. Februar 1892 wurden die drei Fadenschlingen frei in der Scheide liegend gefunden. Das Befinden der Kranken war fortdauernd ein ausgezeichnetes.

Der eben geschilderte Fall ist in mehrfacher Beziehung interessant; erstens durch das Auftreten einer Inversion bei einer 78jährigen Frau, welche 58 Jahre vorher einmal geboren und 30 Jahre bereits im Klimakterium stand. Nach den Angaben dieses Falles kann wohl nicht angenommen werden, dass die Inversion. vielleicht puerperalen Ursprunges gewesen, denn die Frau hatte sich ja in diesen 58 Jahren, seit ihrer einzigen Entbindung, vollkommen wohl befunden, weder an Blutungen, noch an Ausfluss gelitten und erst im letzten Jahre, bevor sie in unsere Beobachtung kam, hatten sich Schmerzen, Urinbeschwerden, leichte Blutabgänge und vermehrte Absonderung eingestellt und war ganz allmälig jener früher beschriebene Körper aus der Vulva zum Vorschein gekommen, wobei allerdings nicht ausgeschlossen ist, dass die bereits begonnene Inversion und der Vorfall des invertirten Uterus durch Zerren seitens der Kranken vervollständigt und vergrössert worden war.

Wollen wir uns in diesem Falle die Frage nach der Entstehung der Inversion vorlegen, so wird es sich als zweckmässig herausstellen, den heute maassgebenden Ansichten über die Entstehung der puerperalen und nicht puerperalen Inversion etwas nachzugehen. Die Aetiologie der puerperalen Inversion ist wohl zweifellos feststehend. Es handelt sich in jedem solchen Falle zunächst um hochgradige Erschlaffung des Uterus; dazu kommt Druck von oben oder $\mathrm{Zug}$ von unten, damit Inversion entstehen könne. Nicht so klar gestellt ist jedoch die Entstehung der nicht puerperalen Inversion. Wir treffen hier auf verschiedene Ansichten. Das Eigenthümliche und Gemeinsame fast aller Fälle 
von nicht puerperaler Inversion ist das gleichzeitige Vorhandensein eines kleineren oder grösseren, mehr weniger gestielt aufsitzenden Myoms in der Nähe des Fundus uteri. Es geht nun die Vorstellung einiger Forscher dahin, dass durch das Wachsthum eines solchen, im Fundus entwickelten Tumors in die Höhle der Cervix und aus dem Muttermunde heraus der Fundus nachgezogen werde und auf diese Weise die Inversion zu Stande käme. Nach Scanzoni soll es an der Basis des Tumors ausserdem noch zur Verdünnung und Verfettung der Muskulatur kommen, durch welche Veränderungen die Wand erschlafft und für die Inversion vorbereitet würde.

Nach Fritsch sollen die Vorbedingungen einer nicht puerperalen Inversion gegeben sein in stärkerer Hyperämie, entzündlicher Erweichung des Uterus und Wachsthum eines Myoms in die untere Uterushälfte, dadurch Erweiterung des Muttermundes, wobei schliesslich durch Zusammenziehung das tiefertretende Myom die Uteruswand als Stiel nach sich ziehen soll.

Pozzi stellt sich vor, dass die Inversion dadurch entstehe, dass ein Theil des Uterus gelähmt sei und durch den uber ihm gelegenen Abschnitt des Uterusmuskels bei dessen Zusammenziehung nach Art eines Schluckactes eingestülpt werde.

Nach Martin entsteht die Inversion so, dass Myome, die polypös geworden, Uteruscontractionen anregen und schliesslich geboren werden, während sie an ihrem Stiele den Uterusfundus nach sich ziehen.

Emmet schreibt die Inversion der Wirkung unregelmässiger theilweiser Muskelzusammenziehungen zu.

Nach Winckel kommt die Inversion in der Weise zu Stande, dass ein breit aufsitzendes submuköses Myom die Uterushöhle, hierauf den inneren Muttermund erweitert, ebenso den Cervicalcanal, und endlich von dem dasselbe umfassenden Uterus herabgezerrt werde und schliesslich theils durch sein eigenes Gewicht, zum Theil durch Contractionen die Stelle der Wand, an der das Myom sitzt, herabziehe.

Nach Schroeder kommt die Inversion bei breit aufsitzenden submukösen oder interstitiellen Myomen, bei schlaffer Uteruswand durch die Schwere des Tumors, der die Cervix auseinanderdrängt, und durch den intraabdominalen Druck allmälig zu Stande. In manchen Fällen bilde sich auch wohl, wenn die in die Uterushöhle 
hereinsinkende Geschwulst eine gewisse Tiefe erreicht hat, die Inversion schnell unter Contractionen.

Wenn wir diese verschiedenen Ansichten der Forscher einer Kritik unterziehen, so werden wir am besten vorgehen, wenn wir die feststehende und bekannte Thatsache bei der Entstehung der puerperalen Inversion uns vor Augen halten. Die nie fehlende Bedingung für die Entstehung einer puerperalen Inversion ist hochgradige Erschlaffung der Uterusmuskulatur. Wenn der Uterus nicht erschlafft ist, wenn er sich contrahirt, so entsteht nie eine Inversion, weder durch Druck von oben, noch durch Zug nach unten. Die Ausdehnung seiner Höhle, das Herabtreten der theilweise gelösten, theilweise noch anhängenden Placenta durch den Muttermund wird nie eine Inversion hervorrufen, wenn nicht der Uterusmuskel sich in einem hochgradigen Erschlaffungszustande befindet. Auch durch Druck von oben wird es bei contrahirtem Uterus, auch mit der grössten Kraftanwendung, nicht gelingen, einen Uterus einzustülpen. Wenn wir also für die Entstehung der puerperalen Inversion Erweiterung der Höhle, Erschlaffung der Uteruswand als Vorbedingung fordern müssen und die Inversion durch Druck von oben oder durch Zug von unten zu Stande kommt, so werden wir nicht fehlgehen, wenn wir dieselben Vorbedingungen und veranlassenden Ursachen auch für die nicht puerperale Inversion fordern.

Die Erweiterung der Uterushöhle bei nicht puerperaler Inversion wird gegeben durch ein in die Uterushöhle hineinwachsendes mehr weniger gestieltes Myom, die Erschlaffung theils durch Atrophie, theils durch entzündliche Infiltration oder Degeneration der Muskulatur. Sind diese Vorbedingungen gegeben, so wird bei Anstrengung der Bauchpresse, also durch Druck von oben, die Inversion allmälig in der Weise zu Stande kommen können, dass sich in dem weichen bildsamen Organe durch den intraabdominalen Druck, vielleicht gerade an der Stelle der grössten Verdünnung, also dort, wo das Myom aufsitzt, eine Delle bildet, welche dann unter der weiteren Wirkung des intraabdominalen Druckes mehr und mehr vertieft wird und erst zur theilweisen und weiterhin zur vollständigen Inversion führen kann. Zweitens kann aber auch durch die Schwere des Tumors allein, also durch Zug von unten, eine Inversion bei schlaffem Uterusmuskel zu Stande kommen; in der Regel werden sich aber bei nicht puerperaler Inversion diese beiden Ursachen mit einander verbinden, indem der Druck von oben und der Zug von unten durch die Schwere 
des Tumors sich zur schliesslichen Wirkung der Einstülpung vereinen. Zusammenziehungen des Uterus können bei der Entstehung der nicht puerperalen Inversion ebensowenig eine Rolle spielen, wie bei der Entstehung der puerperalen Einstülpung; denn sind sie schwach, so können sie keine Inversion zu Stande bringen, sie werden dann auf die Vorwärtsbewegung des in der Uterushöhle liegenden Myoms keine Einwirkung üben können, und nur mit der Vorwärtsbewegung des Myoms wird ja die allmälige Einstülpung der Wand erklärt, indem die Forscher sich vorstellen, das Myom ziehe den Uterus gewissermaassen als Stiel nach sich. Starke Zusammenziehungen jedoch, welche im Stande wären, jene Ortsveränderungen des Myoms hervorzurufen, werden gleichzeitig eine Formveränderung des Uterus verhindern. Denn nur der nicht contrahirte Muskel ist weich und biegsam, der contrahirte Muskel ist starr und unnachgiebig, und es kann also Contraction und Formveränderung des Muskels nicht gleichzeitig oder gar eines als Folge des anderen auftreten. Contrahirt sich der Uterus, so kann er allerdings auf die Vorwärtsbewegung des Myoms einen Einfluss ausüben, aber nicht in der Weise, dass das Myom sich vorwärts bewegt und den Uterus nach sich zieht, sondern nur in der Weise, dass das sich vorwärts bewegende Myom seinen $\mathrm{Zu}$ sammenhang mit der starren Uteruswand lockert und sich allmälig immer mehr abstielt. Wäre das nicht der Fall, so müsste eigentlich in jedem Falle von gestielten, von der Uteruswand entspringenden polypösen Myomen, die wir in die Scheide geboren finden, eine Inversion bestehen. Die Erfahrung lehrt aber, dass diese Inversionen infolge von Myomen zu den grossen Seltenheiten gehören, und es müssen also ganz besondere Bedingungen vorhanden sein, welche die Abwickelung der Vorgänge gestatten, welche bei gestielten Myomen zur Inversion führen.

Auch in unserem Falle ergab die mikroskopische Untersuchung hochgradige Atrophie des Uterus und entzündliche Infiltration seiner Wandungen. Das schlaffe morsche Organ der alten Frau war gewiss einer ausgiebigen Contraction nicht mehr fähig; aber eben aus diesem Grunde kam es bei der Beschäftigung der Frau zur Inversion. Besonders wenn wir bedenken, dass die Kranke ein etwa wallnussgrosses Myom dicht hinter der rechten Tubenmündung aufsitzend trug, dass sie durch ihren Beruf gezwungen war, täglich viele Stunden zu stehen, und dass sie ausserdem, wie von ihr selbst und ihren Anverwandten bestimmt versichert wurde, seit längerer Zeit an hartnäckiger Obstipation litt. 
Ich halte also die Ansicht der meisten Forscher, der zufolge die Inversion dadurch zu Stande kommt, dass durch Zusammenziehungendes Uterusdas Myom geboren wird und den Uterus als Stiel nach sich ziehe, für unrichtig, denn durch die Contraction kann nicht gleichzeitig eine Inversion zu Stande kommen. Zur Inversion gehört immer eine Erschlaffung des Muskels, und uiberall dort, wo wir Inversion finden, müssen wir eine solche annehmen, und wir thun dies auch, wie z. B. beim gleichen Vorgange der Invagination des Darmes. Ich glaube, dass wir für die Entstehung der nicht puerperalen Inversion keine anderen mechanischen Kräfte heranzuziehen brauchen, als für die Entstehung der puerperalen, denn sonst müssten wir uns auch vorstellen können, dass auch bei einer am Fundus theilweise festsitzenden Placenta durch heftige Zusammenziehungen die Placenta geboren würde und den Uterus als Stiel nach sich ziehe, was in Wirklichkeit doch nie vorkommt und unseren Vorstellungen direct zuwider läuft.

Von allen den Forschern scheint nur Schröder eine ähnliche Entstehung der Inversion, wie ich sie eben dargelegt, anzunehmen, und doch glaubt auch er, dass unter Umständen eine Inversion unter Contractionen schnell zu Stande kommen könne, was ich nicht für möglich halte.

Was die Technik der operativen Entfernung des Uterus in solchen Fällen betrifft, so scheint mir die von mir geübte Operationsmethode durch ihre ausserordentliche Einfachheit den Vorzug vor allen anderen zu verdienen. Zunächst halte ich nicht für nothwendig, in diesen Fällen durch typische Totalexstirpation die Operation zu compliciren und zu erschweren, da ja das ganze Organ durch die Umstülpung zugänglich geworden und wir ja nichts $\mathrm{zu}$ thun brauchen, als die im Inversionstrichter liegenden Ligamente mit ihren Gefässen zu versorgen, um dann sofort das Organ durch Amputation entfernen zu können. Wenn Kaltenbach zunächst die Basis des Tumors durch eine Gummischnur abschnürt, dann den Uteruskörper abträgt und das Peritoneum vernäht, so würde ich fürchten, dass trotz der Gummischnur nach der Durchtrennung die Ligamente sich zurückziehen könnten ein Ereigniss, das von den unangenehmsten Folgen begleitet sein könnte.

Schülein legt durch den Rand der Cervix und den obersten Theil des invertirten Uterus Seidenschlingen, welche eine Zurück- 
stülpung des Stumpfes nach der Abtragung verhindern sollen, knotet dieselben jedoch erst nach der Amputation. Auch bei dieser Methode scheint mir das Zurückschlüpfen der stark gespannten Ligamente nicht vollkommen verhindert, und ich ziehe es deshalb vor, durch eine fortlaufende Partienligatur die Gefässe zu unterbinden, die Ligamente am Stumpfe sicher zu befestigen und darunter abzutragen - eine Operation, welche in 5 Minuten bequem ausführbar ist. Gerade in unserem Falle, bei der senilen atrophischen Person, schien mir der Vortheil einer raschen Operation sebr in die Augen zu springen. 


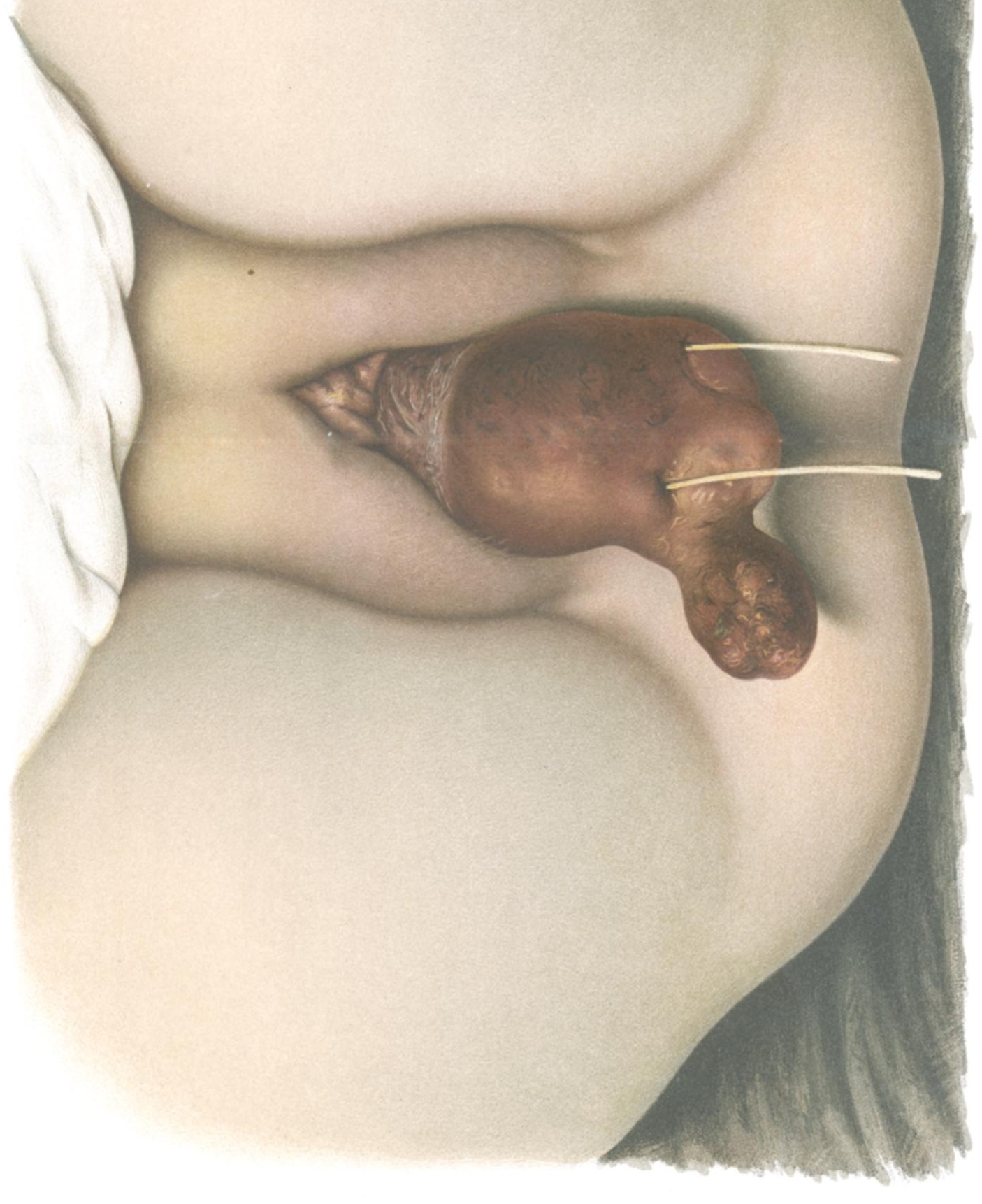

